\title{
HOMOLOGICAL DIMENSION IN LOCAL RINGS
}

\author{
BY \\ MAURICE AUSLANDER AND DAVID A. BUCHSBAUM( ${ }^{(1)}$
}

Introduction. This paper is devoted primarily to the study of cominutative noetherian local rings. The main task is to compare purely algebraic properties with properties of a homological nature. A large part of this paper is an elaboration of [2] $\left({ }^{2}\right)$ which contained no proofs. We use [3] as a reference source for homological algebra. We begin with a list of the most important notions and an outline of results.

Unless stated otherwise, we assume throughout this paper that all rings are commutative noetherian rings with identity elements and that all modules are finitely generated and unitary. If $R$ is a local ring, we shall denote its maximal ideal by $\mathfrak{m}$ and the quotient field $R / \mathfrak{m}$ by $F$.

Given a ring $R$ and an $R$-module $E$ we denote by $\operatorname{hd}_{\mathrm{R}} E$ the integer (finite or $+\infty)$ which in [3] is denoted by $\operatorname{dim}_{R} E$. We have hd $\mathrm{d}_{\mathrm{R}} E \leqq n$ if there exists an exact sequence

$$
0 \rightarrow P_{n} \stackrel{d_{n}}{\longrightarrow} P_{n-1} \stackrel{d_{n-1}}{\longrightarrow} \cdots \rightarrow P_{0} \rightarrow E \rightarrow 0
$$

where each $P_{i}$ is projective (i.e., is a direct summand of a free $R$-module). With the ring $R$ we associate two numbers called the global dimension and the finitistic global dimension given by the definitions

$$
\text { gl. } \operatorname{dim} R=\text { sup. } \operatorname{hd}_{\mathrm{R}} E \quad \text { for all } R \text {-modules } E
$$

f. gl. $\operatorname{dim} R=$ sup. hd $\mathrm{d}_{\mathrm{R}} E \quad$ for all $R$-modules $E$ such that $\mathrm{hd}_{\mathrm{R}} E<\infty$.

By [I, Theorem 1] we know that the global dimension as defined above gives the same number as the definition of global dimension given in [3]. If $R$ is a local ring, then by $\left[3, \mathrm{VIII}, 6.2^{\prime}\right]$ we have that $\mathrm{gl}$. $\operatorname{dim} R=\mathrm{hd}_{\mathrm{R}} F$.

Let $\mathfrak{p}$ be a prime ideal in $R$. We define the rank of $p$ (notation: rank $\mathfrak{p}$ ) as the maximum $n$ for which there exists a sequence $p_{0} \subset p_{1} \subset \cdots \subset p_{n}=p$ of distinct prime ideals in $R$. It is well known that the rank of a prime ideal is always finite. The rank of an ideal $\mathfrak{t}$ in $R$ (notation: rank $\mathfrak{t}$ ) is defined as the min rank $\mathfrak{p}$, where the $\mathfrak{p}$ run through the primes belonging to $R$ in $R$. The Krull dimension of $R$ (notation: $\operatorname{dim} R$ ) is defined as the sup rank $\mathfrak{f}$, where $\mathfrak{f}$ runs through all ideals in $R$.

Received by the editors December 5, 1955 and, in revised form, April 6, 1956 and July 5, 1956.

(1) Some of this work done while one of the authors (Buchsbaum) was a National Science Foundation Postdoctoral Research Fellow at Princeton University.

(2) Numbers in brackets refer to the bibliography at end of paper. 
An element $x$ in $R$ is said to be a zero divisor for the $R$-module $E$ if there is a nonzero element $e$ in $E$ such that $x e=0$. A sequence $x_{1}, \cdots, x_{8}$ of elements in $R$ is called an $E$-sequence if $x_{i}$ is not a zero divisor for the module $E /\left(x_{1}, \cdots, x_{i-1}\right) E$ and $E /\left(x_{1}, \cdots, x_{s}\right) E \neq 0$. The least upper bound of lengths of $E$-sequences (finite or $+\infty$ ) is called the codimension of $E$ (notation: $\left.\operatorname{codim}_{R} E\right)$.

Assume now that $R$ is a local ring. It is well known that

$$
\operatorname{dim} R=\operatorname{rank} \mathfrak{m} \leqq\left(\mathfrak{m} / \mathfrak{m}^{2}: F\right)
$$

where the right hand number is the linear dimension of the vector space $\mathfrak{m} / \mathfrak{m}^{2}$ over the field $F$. If equality holds, then $R$ is called a regular local ring.

The various numbers attached to the local ring $R$ compare as follows:

f. gl. $\operatorname{dim} R=\operatorname{codim}_{R} R \leqq \operatorname{dim} R \leqq\left(\mathfrak{m} / \mathfrak{m}^{2}: F\right) \leqq$ gl. $\operatorname{dim} R$.

The first two relationships will be established in the first section of the paper. The next inequality was stated above. The last inequality was proved by Serre [10] who showed that if $n=\left(\mathfrak{m} / \mathfrak{m}^{2}: F\right)$, then $\operatorname{Tor}_{n}^{R}(F, F) \neq 0$.

It will be shown that $R$ is a regular ring if and only if $\operatorname{gl} \operatorname{dim} R<\infty$, i.e., if and only if all the above inequalities are equalities. Using this result we show that if $R$ is a regular local ring, then $R_{\mathfrak{p}}$ is a regular local ring for any prime ideal $\mathfrak{p}$ in $R$.

$\$ 2$ is devoted to a study of regular local rings. It is shown that any factor ring of a regular local ring (consequently any complete local ring) satisfies the "saturated chain" condition for prime ideals. Also another proof of the Cohen-Macaulay theorem is given.

In $\S 3$, we prove certain properties about the completion of Zariski rings. In particular, we show that if $R$ is a local ring, and $\hat{R}$ its completion, then gl. $\operatorname{dim} R=$ gl. $\operatorname{dim} \hat{R}$. Using this fact, together with the fact that every complete local ring is the quotient of a regular local ring, we prove theorems on $\operatorname{codim}_{R} E$ analogous to those proved in $\S 1$.

In $\S 4$, we introduce the notion of a regular ring, naturally extending the definition from local rings to arbitrary noetherian rings. For these rings, we show that the Cohen-Macaulay theorem is valid, and we also show that a commutative noetherian hereditary ring is the direct sum of a finite number of Dedekind rings.

1. Codimension. Let $R$ be any commutative ring with identity element (not necessarily noetherian) and $S$ a multiplicatively closed subset of $R$ not containing 0 . Then we denote by $R_{S}$ the ring of quotients of $R$ with respect to $S$ [see $\S 9$, no. 48]. If $\mathfrak{p}$ is a prime ideal in $R$, and $S=R-\mathfrak{p}$, then we will denote $R_{S}$ by $R_{\mathfrak{p}}$. The natural ring homomorphism $R \rightarrow R_{S}$, gives an operation of $R$ on $R_{S}$ which makes $R_{S}$ an $R$-module. It has been shown in $[9$, no. 48$]$ that for any $R$-module $E$ ( $E$ not necessarily finitely generated), $\operatorname{Tor}_{n}^{R}\left(R_{S}, E\right)=0$ for all $n>0$. Hence $R_{S} \otimes_{R} E$ is an exact functor of $E$. Thus, if $E^{\prime}$ is a submodule 
of $E$, then $R_{S} \otimes_{R} E^{\prime}$ is a submodule of $R_{S} \otimes_{R} E$.

Suppose now that $R$ is a noetherian ring and that $E$ is a finitely generated $R$-module. We recall that for the submodules of $E$ there exists a primary decomposition theory analogous to that for ideals (see [8]). A prime ideal $\mathfrak{p}$ in $R$ is said to belong to a submodule $E^{\prime}$ of $E$ if $p$ belongs to the annihilator in $R$ of $E / E^{\prime}$. In the following lemma, which we state without proof, we summarize the part of this theory that we shall need.

LEMMA 1.1. Let $S$ be a multiplicatively closed subset of $R$ not containing 0 , and let $E^{\prime}$ be a submodule in the $R$-module $E$. Then $p_{1}, \cdots, p_{n}$, the prime ideals in $R$ belonging to $E^{\prime}$ in $E$, have the following properties:

(a) An element $x$ in $R$ is in one of the $\mathfrak{p}_{i}$ if and only if $x$ is a zero divisor for $E / E^{\prime}$;

(b) An ideal in $R$ is contained in one of the $\mathfrak{p}_{i}$ if and only if there is an element e in $E$ which is not in $E^{\prime}$ such that $\mathfrak{l} e$ is contained in $E^{\prime}$;

(c) Let $\mathfrak{p}_{1}, \cdots, \mathfrak{p}_{r}$ be those ideals which do not meet $S$. Then $E^{\prime} \otimes_{R} R_{S}$ is a proper submodule of the $R_{S}$-module $E \otimes_{R} R_{S}$ if and only if $r>0$. If $r>0$, then $R_{S} p_{1}, \cdots, R_{S} p_{r}$ are the prime ideals in $R_{S}$ belonging to $E^{\prime} \otimes_{R} R_{S}$ in $E \otimes_{R} R_{S}$.

Lemma 1.2. Let $E$ be an $R$-module and let $p^{\prime \prime}$ be a prime ideal in $R$ which belongs to (0) in E. If $x$ is an element in $R$ such that the ideal $\left(\mathfrak{p}^{\prime \prime}, x\right)$ is $\neq R$, then $E \neq x E$. Further, if $x$ is not a zero divisor of $E$, and $\mathfrak{p}$ is a proper prime ideal containing $\left(\mathfrak{p}^{\prime \prime}, x\right)$, then there is a prime ideal $\mathfrak{p}^{\prime}$ belonging to $(0)$ in $E / x E$ such that $\mathfrak{p} \supset \mathfrak{p}^{\prime} \supset\left(\mathfrak{p}^{\prime \prime}, x\right)$.

Proof. Since $\left(\mathfrak{p}^{\prime \prime}, x\right)$ is a proper ideal of $R$, there exists a proper prime ideal $\mathfrak{p}$ in $R$ which contains $\left(\mathfrak{p}^{\prime \prime}, x\right)$. From the exact sequence of $R$-modules

$$
E \stackrel{f}{\rightarrow} E \rightarrow E / x E \rightarrow 0
$$

we deduce the exact sequence of $R_{\mathfrak{p}}$-modules

$$
E \otimes_{R} R_{\mathfrak{p}} \stackrel{g}{\rightarrow} E \otimes_{R} R_{\mathfrak{p}} \rightarrow(E / x E) \otimes_{R} R_{\mathfrak{p}} \rightarrow 0,
$$

where $f$ is multiplication by $x$ in $R$ and $g$ is multiplication by the image of $x$ in $R_{\mathfrak{p}}$ which we shall denote also by $x$. Since $\mathfrak{p}^{\prime \prime} \subset \mathfrak{p}$, and $\mathfrak{p}^{\prime \prime}$ is a prime belonging to $(0)$ in $E$, we have by 1.1 that $E \otimes_{R} R_{\mathfrak{p}} \neq(0)$. Since $x$ is in $R_{\mathfrak{p} p}$, the maximal ideal of the local ring $R_{\mathfrak{p}}$, we know by [3, VIII, Proposition 5.1'] that $g$ is not an epimorphism. Therefore $(E / x E) \otimes_{R} R_{\mathfrak{p}} \neq 0$, which means that $E / x E \neq 0$.

Suppose $x$ in $R$ is not a zero divisor for $E$. Then it can easily be seen that $x$ in $R_{\mathfrak{p}}$ is not a zero divisor for $E \otimes_{R} R_{\mathfrak{p}}$. Since $\mathfrak{p}^{\prime \prime}$ belongs to (0) in $E$, we have by 1.1 that $R_{\mathfrak{p}} \mathfrak{p}^{\prime \prime}$ belongs to (0) in $E \otimes_{R} R_{\mathfrak{p}}$. Therefore we know that there is a prime ideal $\mathfrak{p}$ in the local ring $R_{\mathfrak{p}}$ such that $\mathfrak{p}$ belongs to $(0)$ in $\left(E \otimes_{R} R_{\mathfrak{p}}\right) / x\left(E \otimes_{R} R_{\mathfrak{p}}\right)$ and $R_{\mathfrak{p}} \mathfrak{p} \supset \mathfrak{p} \supset\left(R_{\mathfrak{p}} \mathfrak{p}^{\prime \prime}, x\right)=R_{\mathfrak{p}}\left(\mathfrak{p}^{\prime \prime}, x\right)$ (see $[5,135$ no. 8]; also [10, Lemma 1$])$. From the exact sequence (1) we deduce that 


$$
\left(E \otimes_{R} R_{\mathfrak{p}}\right) / x\left(E \otimes_{R} R_{\mathfrak{p}}\right) \approx(E / x E) \otimes_{R} R_{\mathfrak{p}} .
$$

Since $(E / x E) \otimes_{R} R_{\mathfrak{p}} \neq 0$, we have by 1.1 that $\overline{\mathfrak{p}}=R_{\mathfrak{p}} \mathfrak{p}^{\prime}$, where $\mathfrak{p}^{\prime}$ is a prime belonging to $(0)$ in $E / x E$ which is contained in $\mathfrak{p}$. Thus by contraction to $R$ we have $\mathfrak{p} \supset \mathfrak{p}^{\prime} \supset\left(\mathfrak{p}^{\prime \prime}, x\right)$. Since $\mathfrak{p}$ was any proper prime ideal containing $\left(\mathfrak{p}^{\prime \prime}, x\right)$, the lemma has been established.

Unless stated otherwise, we assume throughout the rest of this section that $R$ is a local ring with maximal ideal $\mathfrak{m}$, and $F=R / \mathfrak{m}$.

Proposition 1.3. Let $p_{1}, \cdots, p_{n}$ be the prime ideals in $R$ belonging to (0) in E. If $x_{1}, \cdots, x_{s}$ is an E-sequence, then $\left(x_{1}, \cdots, x_{s}\right)$ is contained in $\mathfrak{m}$ and $s \leqq \operatorname{dim} R / \mathfrak{p}_{i}$ for all $i$. Thus we have that $\operatorname{codim}_{R} E \leqq \operatorname{dim} R<\infty$.

Proof. Suppose $x_{i}$ is not in $\mathfrak{m}$ for some $i$. Then $x_{i}$ is a unit in $R$ and thus $x_{i} E=E$. Since $\left(x_{1}, \cdots, x_{s}\right) E$ contains $x_{i} E=E$, we have that $E /\left(x_{1}, \cdots, x_{s}\right) E$ $=0$, which contradicts the definition of an $E$-sequence.

Let $\mathfrak{p}$ be one of the $\mathfrak{p}_{i}$. We will show by induction that $s \leqq \operatorname{dim} R / \mathfrak{p}$. For $s=0$, it is obvious. Assume the statement true for $s-1$. By 1.2 , we know there is a prime $\mathfrak{p}^{\prime}$ belonging to $(0)$ in $E / x_{1} E$ with $\mathfrak{p}^{\prime} \supset\left(\mathfrak{p}, x_{1}\right)$.

Now $x_{2}, \cdots, x_{s}$ is an $E / x_{1} E$-sequence. Therefore we have by our induction hypothesis that $s-1 \leqq \operatorname{dim} R / \mathfrak{p}^{\prime}$. Since $x_{1}$ is not contained in the prime ideal $\mathfrak{p}$, we have that $\operatorname{dim} R / \mathfrak{p}^{\prime} \leqq \operatorname{dim} R / \mathfrak{p}-1$. From this it follows that $s \leqq \operatorname{dim} R / \mathfrak{p}$.

The rest of the proposition follows from the definition of codimension and the fact that $\operatorname{dim} R / \mathfrak{l} \leqq \operatorname{dim} R$ for any ideal $\mathfrak{l}$ in $R$.

PROPOSITION 1.4. If $x_{1}, \cdots, x_{s}$ is an $E$-sequence, then $h d_{R} E /\left(x_{1}, \cdots, x_{s}\right) E$ $=s+h d_{R} E$.

Proof. Suppose an element $x$ in $\mathfrak{m}$ is not a zero divisor for $E$. From the exact sequence

$$
0 \rightarrow E \stackrel{f}{\rightarrow} E \rightarrow E / x E \rightarrow 0
$$

where $f$ is multiplication by $x$, we deduce the exact sequence

$$
\cdots \rightarrow \operatorname{Tor}_{n}(E, F) \stackrel{f^{\prime}}{\rightarrow} \operatorname{Tor}_{n}(E, F) \rightarrow \operatorname{Tor}_{n}(E / x E, F) \rightarrow \operatorname{Tor}_{n-1}(E, F) \stackrel{f^{\prime}}{\rightarrow} \ldots
$$

where $f^{\prime}$ is multiplication by $x$. Since $x F=0$, the homomorphisms $f^{\prime}$ are the zero homomorphisms for all $n$. Hence we have, for every $n$, the exact sequences

$$
0 \rightarrow \operatorname{Tor}_{n}(E, F) \rightarrow \operatorname{Tor}_{n}(E / x E, F) \rightarrow \operatorname{Tor}_{n-1}(E, F) \rightarrow 0 .
$$

If $\operatorname{hd}_{\mathrm{R}} E=\infty$, then we know by $\left[3, \mathrm{VIII}, 6.1^{\prime}\right]$ that $\operatorname{Tor}_{n}(E, F) \neq 0$ for all $n$. From (1) we deduce that $\operatorname{Tor}_{n}(E / x E, F) \neq 0$ for all $n$ and thus $\operatorname{hd}_{\mathrm{R}} E / x E=\infty$. If $\operatorname{hd}_{\mathrm{R}} E=p<\infty$, then we have $\operatorname{Tor}_{p}(E, F) \neq 0$ and $\operatorname{Tor}_{n}(E, F)=0$ for all $n>p$. 
We therefore deduce from (1) that $\operatorname{Tor}_{p+1}(E / x E, F) \neq 0$, and $\operatorname{Tor}_{n}(E / x E, F)$ $=0$ for all $n>p+1$, which yields the desired result for $s=1$. The proof for all $s$ follows by induction.

Lемма 1.5. Let $R, T$ be arbitrary rings with identity element (i.e., not necessarily commutative or noetherian), and $f: R \rightarrow T$ a ring homomorphism. If $E$ is an arbitrary (left) R-module (i.e., not necessarily finitely generated), and $\operatorname{Tor}_{n}^{R}(T, E)=0$ for all $n>0$, then $h d_{R} E \geqq h d_{T}\left(T \otimes_{R} E\right)$. Furthermore, if there exists an R-module homomorphism $g: T \rightarrow R$ such that $g f$ is the identity on $R$, then the above inequality is an equality.

Proof. The first statement is essentially contained in the proof of $[3$, VIII, 3.1]. Hence we need only prove the reverse inequality.

For any $R$-module $C$, consider the identifications

$\operatorname{Hom}_{T}\left(T \otimes_{R} E, T \otimes_{R} C\right) \approx \operatorname{Hom}_{R}\left(E, \operatorname{Hom}_{T}\left(T, T \otimes_{R} C\right)\right) \approx \operatorname{Hom}_{R}\left(E, T \otimes_{R} C\right)$. If we replace $E$ by an $R$-projective resolution $X$ of $E$, and pass to homology, we obtain

$$
H\left(\operatorname{Hom}_{T}\left(T \otimes_{R} X, T \otimes_{R} C\right)\right) \approx H\left(\operatorname{Hom}_{R}\left(X, T \otimes_{R} C\right)\right) .
$$

Since $\operatorname{Tor}_{n}^{R}(T, E)=0$ for all $n>0$, we have that $T \otimes_{R} X$ is a $T$-projective resolution of $T \otimes_{R} E$. Therefore we deduce that

$$
\operatorname{Ext}_{T}\left(T \otimes_{R} E, T \otimes_{R} C\right) \approx \operatorname{Ext}_{R}\left(E, T \otimes_{R} C\right) .
$$

Since $g f$ is the identity, $R$ is a direct summand of $T$ as an $R$-module, which implies that $C$ is a direct summand of $T \otimes_{R} C$ as an $R$-module. Therefore, $\operatorname{Ext}_{R}(E, C)$ is a direct summand of $\operatorname{Ext}_{R}\left(E, T \otimes_{R} C\right)$, which proves the reverse inequality.

As an application of 1.5 we prove

Proposition 1.6. Let $R$ be an arbitrary commutative ring with identity element (not necessarily noetherian), $S$ a multiplicatively closed subset of $R$ not containing 0 , and $E$ an arbitrary $R$-module (not necessarily finitely generated). Then $h d_{R} E \geqq h d_{R_{S}}\left(R_{S} \otimes_{R} E\right)$. Further, we have that gl. $\operatorname{dim} R \geqq g l . \operatorname{dim} R_{S}$.

Proof. By $[9$, no. 48] we know that for an arbitrary $R$-module $E$. $\operatorname{Tor}_{n}^{R}\left(R_{S}, E\right)=0$ for all $n>0$. Thus we have by 1.5 that $h_{\mathrm{R}} E \geqq$ hd $_{\mathrm{R}_{\mathrm{S}}}\left(R_{S} \otimes_{R} E\right)$.

Let $\mathfrak{f}$ be an ideal in $R_{S}$. It is well known that $R_{S} / \mathfrak{f} \approx R_{S} \otimes_{R}(R /(\mathfrak{f} \cap R))$. Since gl. $\operatorname{dim} R \geqq \mathrm{hd}_{\mathrm{R}} R /(\mathfrak{f} \cap R) \geqq \operatorname{hd}_{\mathrm{R}_{\mathrm{g}}} R_{S} / \mathfrak{f}$ for all ideals $\mathfrak{f}$ in $R_{S}$, it follows from [1, Theorem 1] that gl. $\operatorname{dim} R \geqq \mathrm{gl} . \operatorname{dim} R_{S}$.

Theorem 1.7. If $R$ is a local ring, then f. $\mathrm{gl}$. $\operatorname{dim} R=\operatorname{codim} R$.

Proof. If $x_{1}, \cdots, x_{n}$ is an $R$-sequence, then

$$
\operatorname{hd}_{\mathrm{R}} R /\left(x_{1}, \cdots, x_{n}\right) R=n+\operatorname{hd}_{\mathrm{R}} R=n \text {. }
$$

Thus $\operatorname{codim} R \leqq \mathrm{f} . \mathrm{gl} . \operatorname{dim} R$. 
Given any $R$-module $E$, we can construct an exact sequence

$$
0 \rightarrow K \rightarrow X \stackrel{g}{\rightarrow} E \rightarrow 0
$$

with $X$ free and $K=\operatorname{Ker} g \subset \mathfrak{m} X$. For let $e_{1}, \cdots, e_{r}$ be a minimal generating system for $E$. Let $X$ be the free $R$-module with basis $x_{1}, \cdots, x_{r}$ and $g$ the homomorphism which sends $x_{i}$ into $e_{i}$. Now $\sum r_{i} x_{i}$ is in $K$ if and only if $\sum r_{i} e_{i}=0$. Suppose $r_{1}$ is not in $\mathrm{m}$. Then $r_{1}$ is a unit. Thus $e_{1}=-r_{1}^{-1}\left(\sum_{i>1} r_{i} e_{i}\right)$, which contradicts the minimality of $e_{1}, \cdots, e_{r}$. Hence $r_{1}$ and similarly all the other $r_{i}$ must be in $\mathrm{m}$. Therefore $K$ is contained in $\mathrm{m} X$.

Assume that $\operatorname{codim} R=0$ and that f. gl. $\operatorname{dim} R>0$. Then there exist modules $E$ with $0<\mathrm{hd}_{\mathrm{R}} E<\infty$. Since in (2) $\mathrm{hd}_{\mathrm{R}} K=\mathrm{hd}_{\mathrm{R}} E-1$, we may choose $E$ such that $\operatorname{hd}_{\mathrm{R}} E=1$. Then $K$ is projective and therefore free [3, VIII, 6.1']. Thus the annihilator of $K$ is trivial which implies that the annihilator of $\mathfrak{m} X$ is trivial. Consequently the annihilator of $m$ is trivial. However since codim $R$ $=0$, every element of $\mathfrak{m}$ is a zero divisor. This means that $\mathfrak{m}$ belongs to (0) and therefore by 1.1 has a nontrivial annihilator. This contradiction shows that f. gl. $\operatorname{dim} R \leqq \operatorname{codim} R$ if $\operatorname{codim} R=0$.

We proceed now by induction. Assume that f. gl. $\operatorname{dim} R \leqq \operatorname{codim} R$ if $\operatorname{codim} R<n$. Also assume that $\operatorname{codim} R=n>0$. Let $x$ in $\mathfrak{m}$ be a nonzero divisor in $R$ and let $R^{\prime}=R / x R$. It is clear that codim $R^{\prime} \leqq n-1$. Further, we have that $\operatorname{hd}_{\mathrm{R}} R^{\prime}=1$.

Let $E$ be an $R$-module with $0<\mathrm{hd}_{\mathrm{R}} E<\infty$. Consider the exact sequence (2). Since $x$ is not a zero divisor in $X$ (because $X$ is free) it is not a zero divisor in $K \subset X$. Thus $x$ is a $K$-sequence and by 1.4 we know that

$$
\operatorname{hd}_{\mathrm{R}} K / x K=1+\operatorname{hd}_{\mathrm{R}} K=\operatorname{hd}_{\mathrm{R}} E .
$$

Thus if we define $K^{\prime}=K / x K=K \otimes_{R} R^{\prime}$, we have an $R^{\prime}$-module $K^{\prime}$ with $\operatorname{hd}_{\mathrm{R}} K^{\prime}=\mathrm{hd}_{\mathrm{R}} E<\infty$.

Since $\operatorname{hd}_{\mathrm{R}} R^{\prime}=1$, we have $\operatorname{Tor}_{q}^{R}\left(E, R^{\prime}\right)=0$ for $q>1$. The exact sequence (2) then implies $\operatorname{Tor}_{q}^{R}\left(K, R^{\prime}\right)=0$ for $q>0$. Thus by 1.5 we have that

$$
\operatorname{hd}_{R^{\prime}} K^{\prime}=\operatorname{hd}_{R^{\prime}}\left(R^{\prime} \otimes_{R} K\right) \leqq \operatorname{hd}_{\mathrm{R}} K<\infty .
$$

Since $\operatorname{codim} R^{\prime} \leqq n-1$, it follows from the induction assumption that

$$
\operatorname{hd}_{\mathbf{R}^{\prime}} K^{\prime} \leqq n-1 .
$$

We now use the inequality

$$
\operatorname{hd}_{\mathrm{R}} K^{\prime} \leqq \operatorname{hd}_{\mathrm{R}^{\prime}} K^{\prime}+\mathrm{hd}_{\mathrm{R}} R^{\prime}
$$

[3, XVI, Exercise 5]. Substituting previous results in this inequality we obtain that $h_{\mathrm{R}} E \leqq n$. Thus f. gl. $\operatorname{dim} R \leqq \operatorname{codim} R$.

The following result is due to Serre [10, Theorem 4$]$ : 
Proposition 1.8. gl. $\operatorname{dim} R \geqq\left(\mathfrak{m} / \mathfrak{m}^{2}: R / \mathfrak{m}\right)$.

A well known theorem of Krull [7, 3.5, Theorem 7] tells us that $\operatorname{dim} R \leqq\left(\mathfrak{m} / \mathfrak{m}^{2}: R / \mathfrak{m}\right)$.

Summarizing the results of this section we obtain

THEOREM 1.9. If $R$ is a local ring, then

f. $g$ l. $\operatorname{dim} R=\operatorname{codim} R \leqq \operatorname{dim} R \leqq\left(\mathfrak{m} / \mathfrak{m}^{2}: R / \mathfrak{m}\right) \leqq g l . \operatorname{dim} R$.

Theorem 1.10. A necessary and sufficient condition that a local ring $R$ be regular is that gl. $\operatorname{dim} R<\infty$.

Proof. If gl. $\operatorname{dim} R<\infty$, then $\mathrm{gl}$. $\operatorname{dim} R=\mathrm{f}$. gl. $\operatorname{dim} R$. Hence, by Theorem 1.8 , we have that $\operatorname{dim} R=\left(\mathrm{m} / \mathrm{m}^{2}: R / \mathfrak{m}\right)$, so that $R$ is regular.

Now suppose that $R$ is regular. Then it is known that $\mathfrak{m}=\left(u_{1}, \cdots, u_{n}\right)$ where $\left(u_{1}, \cdots, u_{i}\right)$ is a prime ideal for $1 \leqq i \leqq n,\left[7\right.$, p. 72]. Hence $u_{1}, \cdots, u_{n}$ is an $R$-sequence and $\mathrm{gl}$. $\operatorname{dim} R=\mathrm{hd}_{\mathrm{R}} R / \mathfrak{m}=n$.

Combining 1.10 and 1.6 we have

THEOREM 1.11. If $R$ is a regular local ring and $\mathfrak{p}$ is a prime ideal in $R$, then $R_{\mathfrak{p}}$ is a regular local ring.

2. Regular local rings. In this section, all rings will be regular local rings unless otherwise specified.

Proposition 2.1. Let $R$ be an arbitrary local ring (not necessarily regular) and let $E$ be an $R$-module. The E-sequence $x_{1}, \cdots, x_{s}$ is maximal (i.e., there is no $x_{s+1} \in R$ such that $x_{1}, \cdots, x_{s+1}$ is an E-sequence) if and only if $\mathfrak{m}$ belongs to (0) in $E /\left(x_{1}, \cdots, x_{s}\right) E$.

Proof. The $E$-sequence $x_{1}, \cdots, x_{s}$ is maximal if and only if every element of $\mathrm{m}$ is a zero divisor of $E /\left(x_{1}, \cdots, x_{s}\right) E$. By 1.1 that is precisely the condition for $\mathfrak{m}$ to be contained in some prime belonging to $(0)$ in $E /\left(x_{1}, \cdots, x_{s}\right) E$. Since $\mathfrak{m}$ is maximal, this means that $\mathfrak{m}$ belongs to $(0)$ in $E /\left(x_{1}, \cdots, x_{s}\right) E$.

Proposition 2.2. $h d_{R} E=g l$. $\operatorname{dim} R$ if and only if $\mathfrak{m}$ belongs to (0) in $E$.

Proof. Suppose $\mathfrak{m}$ does not belong to $(0)$ in $E$. Then there is an $x$ in $\mathfrak{m}$ which is not a zero divisor of $E$. Therefore, by 1.4 , we have $h_{\mathrm{R}} E / x E$ $=1+\operatorname{hd}_{\mathrm{R}} E$. Since gl. $\operatorname{dim} R$ is finite, this shows that $\mathrm{hd}_{\mathrm{R}} E<\mathrm{gl} . \operatorname{dim} R$.

Now suppose that $\mathfrak{m}$ does belong to (0) in $E$. Then by 1.1 there is a nontrivial submodule $D$ of $E$ such that $\mathrm{m} D=0$. Since $D$ is an $R / \mathrm{m}$-module, $D$ is isomorphic to a finite direct sum of $R / \mathrm{m}$ 's. Therefore we have $\operatorname{Tor}_{n}^{R}(D, F) \neq 0$ where $n=\mathrm{gl}$. $\operatorname{dim} R$. From the exact sequence

$$
0 \rightarrow \operatorname{Tor}_{n}^{R}(D, F) \rightarrow \operatorname{Tor}_{n}^{R}(E, F) \rightarrow \operatorname{Tor}_{n}^{R}(E / D, F) \rightarrow \cdots
$$


we deduce that $\operatorname{Tor}_{n}^{R}(E, F) \neq 0$, which shows that hd ${ }_{R} E=g l . \operatorname{dim} R$.

Combining 1.4, 2.1 and 2.2, we obtain

THEOREM 2.3. If $\operatorname{dim} R=n$, and if $x_{1}, \cdots, x_{s}$ is an E-sequence, then

(a) $s+h d_{R} E \leqq n$,

(b) $s+h d_{R} E=n$ if and only if $x_{1}, \cdots, x_{s}$ is a maximal E-sequence,

(c) any E-sequence can be extended to a maximal E-sequence,

(d) $h d_{R} E+\operatorname{codim}_{R} E=n$.

PROPOSITION 2.4. Let be a proper ideal in $R, R$ a commutative noetherian ring, and let $x_{1}, \cdots, x_{s}$ be an $R / \mathfrak{f}$-sequence. Then

(a) $\operatorname{rank}\left(\mathfrak{l}, x_{1}, \cdots, x_{s}\right) \geqq s+\operatorname{rank} \mathfrak{l}$,

(b) if $\mathfrak{l}=(0)$, then rank $\left(x_{1}, \cdots, x_{s}\right)=s$.

Proof. It suffices to prove (a). For if (a) is established, we have rank $\left(x_{1}, \cdots, x_{s}\right) \geqq s$. However, a well-known result of Krull [7, III, Theorem 7] states that rank $\left(x_{1}, \cdots, x_{s}\right) \leqq s$. Thus (b) follows from (a).

We prove (a) by induction on $s$. Assume $s=1$. Let $p$ be a minimal prime of $\left(\mathfrak{l}, x_{1}\right)$. Since $\left(\mathfrak{l}, x_{1}\right) / \mathfrak{l}$ is a principal ideal in $R / \mathfrak{l}$, generated by a nonzero divisor, it follows from 1.1 and the principal ideal theorem that $\mathfrak{p} / \mathfrak{l}$ is of rank one in $R / \mathfrak{t}$. Therefore rank $\mathfrak{p} \geqq 1+$ rank $\mathfrak{l}$. The inductive procedure is now straightforward.

Proposition 2.5. Let $R$ be a regular local ring and let $E$ be an $R$-module. Then $h d_{R} E \geqq$ rank $\mathfrak{p}$, where $\mathfrak{p}$ is any prime ideal belonging to $(0)$ in $E$.

Proof. We let $n=\operatorname{dim} R$. Then by 2.3 we have that $\operatorname{hd}_{\mathrm{R}} E+\operatorname{codim}_{R} E=n$. If $\mathfrak{p}$ is a prime belonging to (0) in $E$, we have by 1.3 that $\operatorname{codim}_{R} E \leqq \operatorname{dim} R / \mathfrak{p}$. Thus $n \leqq \mathrm{hd}_{\mathrm{R}} E+\operatorname{dim} R / \mathfrak{p}$. It is also clear, however, that $\operatorname{dim} R / \mathfrak{p}+\operatorname{rank} \mathfrak{p} \leqq n$ (we will see later that we actually have equality). Hence we have the desired result.

CoROllaRy 2.6. If $\mathfrak{l}$ is a proper ideal of $R$ and $h d_{R} R / \mathfrak{l}=\operatorname{rank} \mathfrak{l}$, then $\mathfrak{l}$ is unmixed (i.e., all primes belonging to have the same rank).

Proposition 2.7. Let $\mathfrak{l}$ be a proper ideal of $R$, of rank $s$. Then if $x_{1}, \cdots, x_{\mathfrak{t}}$ is any $R$-sequence in $\mathfrak{l}$ such that there is no $x_{t+1}$ in $\mathfrak{l}$ for which $x_{1}, \cdots, x_{t+1}$ is an $R$-seguence, then $t=s$. In particular, $\mathfrak{l}$ contains an $R$-sequence $x_{1}, \cdots, x_{s}$.

Proof. Let $x_{1}, \cdots, x_{t}$ be an $R$-sequence in $\mathfrak{l}$ having the property stated in the hypothesis. Then $\mathfrak{l}$ must be contained in some prime belonging to $\left(x_{1}, \cdots, x_{t}\right)$. However, $\operatorname{hd}_{\mathrm{R}} R /\left(x_{1}, \cdots, x_{t}\right)=t=\operatorname{rank}\left(x_{1}, \cdots, x_{t}\right)$, so that by 2.6 all primes belonging to $\left(x_{1}, \cdots, x_{t}\right)$ have rank $t$. Thus rank $\mathfrak{t} \leqq t$. On the other hand, rank $\geqq \operatorname{rank}\left(x_{1}, \cdots, x_{i}\right)=t$. Hence $t=s$.

Proposition 2.8. Let $\mathfrak{p} \supset \mathfrak{p}^{\prime}\left(\mathfrak{p} \neq \mathfrak{p}^{\prime}\right)$ be two prime ideals in $R$ such that no prime ideal is properly contained between them. Then rank $\mathfrak{p}=1+$ rank $\mathfrak{p}^{\prime}$. 
Proof. Suppose that rank $\mathfrak{p}^{\prime}=s$. Then $\mathfrak{p}^{\prime}$ contains an $R$-sequence $x_{1}, \cdots, x_{8}$, and $\left(x_{1}, \cdots, x_{s}\right)$ is an unmixed ideal of rank $s$. Since rank $p>s$ there is an $x_{s+1}$ in $\mathfrak{p}$, such that $x_{1}, \cdots, x_{s+1}$ is an $R$-sequence. By 1.2 we know that there is a prime $\mathfrak{p}^{\prime \prime}$ belonging to $\left(x_{1}, \cdots, x_{s+1}\right)$ such that $\mathfrak{p} \supset \mathfrak{p}^{\prime \prime} \supset\left(\mathfrak{p}^{\prime}, x_{s+1}\right) \supset \mathfrak{p}^{\prime}$ $\left(\mathfrak{p}^{\prime}, x_{s+1} \neq \mathfrak{p}^{\prime}\right)$. Therefore $\mathfrak{p}=\mathfrak{p}^{\prime \prime}$. Since $\left(x_{1}, \cdots, x_{s+1}\right)$ is unmixed and of rank $s+1$, we have rank $\mathfrak{p}=s+1=1+\operatorname{rank} \mathfrak{p}^{\prime}$.

The following corollaries are immediate consequences of 2.8 and 1.9.

COROllary 2.9. If $\mathfrak{p}$ is a prime ideal in $R$, then $\operatorname{dim} R / \mathfrak{p}+\operatorname{rank} \mathfrak{p}=\operatorname{dim} R$.

COROLlaRy 2.10. If $R$ is a factor ring of a regular local ring, and $p \supset \mathfrak{p}^{\prime}$ are two prime ideals of $R$, then all saturated chains of prime ideals between $\mathfrak{p}$ and $\mathfrak{p}^{\prime}$ have the same length, namely $\operatorname{dim} R / \mathfrak{p}^{\prime}-\operatorname{dim} R / \mathfrak{p}$.

LeMma 2.11. Let $R$ be a commutative noetherian ring having the property that if $p \supset \mathfrak{p}^{\prime}$ are prime ideals of $R$, then any two saturated chains of prime ideals between $\mathfrak{p}$ and $\mathfrak{p}^{\prime}$ have the same length (we will say that such a ring $R$ satisfies the "saturated chain condition"). If $\mathfrak{f}$ is any ideal of $R$ and $x$ is a nonunit in $R$, then $\operatorname{rank}(\mathfrak{f}, x) \leqq 1+\operatorname{rank} \mathfrak{f}$.

Proof. Let rank $\mathfrak{f}=s$, and let $p$ be a prime of rank $s$ belonging to $f$. Then in the ring $R / \mathfrak{p}$, the ideal $(\mathfrak{p}, x) / \mathfrak{p}$ has rank at most one. It follows from the saturated chain condition in $R$ that the ideal $(\mathfrak{p}, x)$ has rank at most $s+1$. Since $(\mathfrak{p}, x)$ contains $(\mathfrak{l}, x)$, we have rank $(\mathfrak{f}, x) \leqq 1+s$.

We can now prove the following slight refinement of the Cohen-Macaulay Theorem:

THEOREM 2.12. Let $\mathfrak{f}=\left(x_{1}, \cdots, x_{8}\right)$ be an ideal of rank $s$. Then $x_{1}, \cdots, x_{8}$ is an $R$-sequence and thus $\mathfrak{t}$ is unmixed.

Proof. By induction on $s$. If $s=1$, then $\mathfrak{f}=\left(x_{1}\right)$ and thus by 2.6 we have that the theorem is true. Consider the ideal $\left(x_{1}, \cdots, x_{s-1}\right), s>1$. Since rank $\mathfrak{f}=s$, it follows from 2.11 that rank $\left(x_{1}, \cdots, x_{s-1}\right) \geqq s-1$. But clearly the reverse inequality holds, since $x_{1}, \cdots, x_{s-1}$ is generated by $s-1$ elements. Applying the induction hypothesis, we have that $x_{1}, \cdots, x_{s-1}$ is an $R$ sequence and thus $x_{1}, \cdots, x_{s-1}$ is unmixed. Since $f$ has rank $s, x_{s}$ cannot be contained in any of the primes belonging to $\left(x_{1}, \cdots, x_{s-1}\right)$. Therefore $x_{1}, \cdots, x_{s}$ is an $R$-sequence, and consequently unmixed.

Corollary 2.13. Let $x_{1}, \cdots, x_{s}$ be an $R$-sequence and let $\sigma$ be a permutation of $1, \cdots, s$. Then $x_{\sigma(1)}, \cdots, x_{\sigma(s)}$ is an R-sequence.

THEOREM 2.14. A regular local ring of dimension two is a unique factorization domain.

Proof. Since a regular local ring is integrally closed in its field of quotients, it suffices to prove that every minimal prime of $R$, i.e., every prime of rank one, is principal. If $\mathfrak{p}$ is a prime ideal of rank one, then $\mathfrak{m}$ does not belong to 
$\mathfrak{p}$ so that $\operatorname{hd}_{\mathrm{R}} R / \mathfrak{p}<\mathrm{hd}_{\mathrm{R}} R / \mathfrak{m}=\mathrm{gl}$. $\operatorname{dim} R=2$. Therefore $\operatorname{hd}_{\mathrm{R}} \mathfrak{p}<1$, hence $\mathfrak{p}$ is projective, therefore free, and therefore principal.

3. Completion. By a Zariski ring, we mean a pair $(R, \mathfrak{l})$ where $R$ is a commutative noetherian ring, and $\mathfrak{l}$ is an ideal contained in the intersection of the maximal ideals of $R$ (in case $R$ is a local ring, we shall always choose $\mathfrak{l}$ to be the maximal ideal $\mathfrak{m}$ ). If $E$ is an $R$-module (finitely generated) we shall denote by $\hat{E}$ the completion of $E$ with respect to $\mathfrak{f}$ (see $[8, \mathrm{~V}]$ ). If $E$ and $E^{\prime}$ are $R$ modules and $f: E \rightarrow E^{\prime}$ is an $R$-homomorphism, then there exists a unique extension $\hat{f}: \hat{E} \rightarrow \hat{E}^{\prime}$ which is an $\hat{R}$-homomorphism. For convenience, we list without proof some of the properties of the completion functor in the following proposition $[8, \mathrm{~V}$, Theorems $4,5,6]$.

Proposition 3.1. (a) If the sequence of $R$-modules

$$
0 \rightarrow E^{\prime} \stackrel{f}{\rightarrow} E \stackrel{g}{\rightarrow} E^{\prime \prime} \rightarrow 0
$$

is exact, then so is the sequence

$$
0 \rightarrow \hat{E}^{\prime} \stackrel{\hat{f}}{\rightarrow} \hat{E} \stackrel{\hat{g}}{\rightarrow} \hat{E}^{\prime \prime} \rightarrow 0
$$

of $\hat{R}$-modules.

(b) If $E^{\prime}$ is a submodule of $E$, then $\hat{E}^{\prime}$ is a submodule of $\hat{E}$ and $\hat{E}^{\prime}=\hat{R} E^{\prime}$, $\hat{E}^{\prime} \cap E=E^{\prime}$.

(c) If $R$ is a local ring with maximal ideal $\mathfrak{m}, \hat{R}$ is a local ring with maximal ideal $\widehat{\mathrm{m}}$.

THEOREM 3.2. If $(R, \mathfrak{t})$ is a Zariski ring, and $E$ is an R-module, then $h d_{R} E \geqq h d_{\widehat{R}} \hat{E}$. If $R$ is a local ring, the above inequality is an equality, and thus gl. $\operatorname{dim} R=g l . \operatorname{dim} \hat{R}$.

Proof. Let $X$ be an $R$-projective resolution of $E$. Then $\hat{X}$ is an acyclic complex over $\hat{E}$, since $E \rightarrow \hat{E}$ is an exact functor. Since the functor is also additive, it takes direct sums into direct sums, hence it takes summands of finitely generated free $R$-modules into summands of finitely generated free $\hat{R}$-modules. Therefore finitely generated projective $R$-modules are carried into finitely generated projective $\hat{R}$-modules. Therefore, $\hat{X}$ is a projective resolution of $\hat{E}$ and $\operatorname{hd}_{\mathrm{R}} E \geqq \mathrm{hd}_{\mathbf{R}} \hat{E}$.

Suppose now that $R$ is a local ring. Then $\hat{R}$ is also a local ring. Let $E$ be an $R$-module with minimal generating system $e_{1}, \cdots, e_{n}$. We will show that this same set is a minimal generating set for $\hat{E}$. Suppose $e_{1}$ is in the $\hat{R}$-module $E^{\prime \prime}$ generated by $e_{2}, \cdots, e_{n}$. Then $E^{\prime \prime}=\hat{E}^{\prime}$ where $E^{\prime}$ is the submodule of $E$ generated by $e_{2}, \cdots, e_{n}$. Since $\hat{E}^{\prime} \cap E=E^{\prime}$, we have $e_{1}$ in $E^{\prime}$, contradicting the minimality of the generating set.

Now, if $\hat{E}$ is $\hat{R}$-projective, it is $\hat{R}$-free, since $\hat{R}$ is a local ring. Therefore $e_{1}, \cdots, e_{n}$ is a free base for $\hat{E}$ over $\hat{R}$. But then this is also a free base for $E$ over $R$. Hence if $\hat{E}$ is $\hat{R}$-projective, then $E$ is $R$-projective. 
Let $E$ be an $R$-module, and $X$ a projective resolution of $E$. Then $\hat{X}$ is an $\hat{R}$-projective resolution of $\hat{E}$. Since kernel $\left(d_{i}\right)$ (where $\left.d_{i}: X_{i} \rightarrow X_{i-1}\right)$ equals the completion of kernel $\left(\hat{d}_{i}\right) \cap X_{i}$, we can deduce that if kernel $\left(\hat{d}_{i}\right)$ is $\hat{R}$ projective, then kernel $\left(d_{i}\right)$ is $R$-projective. Thus $\operatorname{hd}_{\mathrm{R}} E \leqq \mathrm{hd}_{\mathbf{R}} \hat{E}$. The rest of the proposition follows from the fact that the global dimension of a local ring is determined by the homological dimension of its maximal ideal.

Proposition 3.3. Let $(R, \mathfrak{t})$ be a Zariski ring. Then a sequence $x_{1}, \cdots, x_{8}$ of elements in $R$ is an $\hat{E}$-sequence if it is an E-sequence. If $R$ is a local ring, then $x_{1}, \cdots, x_{8}$ is a maximal $\hat{E}$-sequence if it is a maximal E-sequence.

Proof. Let $\mathfrak{l}_{i}$ be the ideal generated by $\left(x_{1}, \cdots, x_{i}\right)$. Since $\mathfrak{l}_{i}=R \cap \hat{\mathfrak{f}}_{i}$, where $\widehat{\mathfrak{f}}_{i}=\hat{R} \hat{\mathfrak{f}}_{i}, \hat{\mathfrak{l}}_{i}$ is a proper ideal of $R$ if and only if $\widehat{\mathfrak{f}}_{i}$ is a proper ideal of $\hat{R}$. From the exact sequence of $R$-modules

$$
E / \mathfrak{t}_{i-1} E \stackrel{f_{i}}{\rightarrow} E / \mathfrak{f}_{i-1} E \stackrel{g_{i}}{\rightarrow} E / \mathfrak{f}_{i} E \rightarrow 0
$$

where $f_{i}$ is multiplication by $x_{i}$ and $g_{i}$ is the natural map, we deduce the exact sequence of $\hat{R}$-modules

$$
\hat{E} / \mathfrak{f}_{i-1} \hat{E} \stackrel{\hat{f}_{i}}{\rightarrow} E / \mathfrak{l}_{i-1} \hat{E} \stackrel{\hat{g}_{i}}{\rightarrow} \hat{E} / \mathfrak{l}_{i} \ddot{E} \rightarrow 0
$$

where, again, $\hat{f}_{i}$ is multiplication by $x_{i}$, and $\hat{g}_{i}$ is natural.

By 3.1 , we know that $\hat{f}_{i}$ is a monomorphism if $f_{i}$ is a monomorphism. Thus, $x_{1}, \cdots, x_{8}$ is an $\hat{E}$-sequence if it is an $E$-sequence.

Now let $R$ be a local ring. Then $m$ belongs to (0) in $E$ if and only if there is a nontrivial submodule $D$ of $E$ such that $\mathrm{m} D=0$. If such a $D$ exists, then $\widehat{\mathrm{m}} \hat{D}=0$, which means that $\widehat{\mathrm{m}}$ belongs to $(0)$ in $\hat{E}$. Therefore, if $x_{1}, \cdots, x_{s}$ is a maximal $E$-sequence in $R$, then $m$ belongs to $(0)$ in $E /\left(x_{1}, \cdots, x_{s}\right) E$ and consequently $\widehat{m}$ belongs to $(0)$ in $\hat{E} /\left(x_{1}, \cdots, x_{s}\right) \hat{E}$, i.e., $x_{1}, \cdots, x_{s}$ is a maximal $\hat{E}$-sequence in $\hat{R}$.

Proposition 3.4. Let $E$ be a nontrivial $R$-module, where $R$ is again a local ring. Then every $E$-sequence can be extended to a maximal E-sequence and all maximal E-sequences have the same length, namely $\operatorname{codim}_{R} E$.

Proof. That every $E$-sequence can be extended to a maximal one follows from 1.2. Hence we need only prove the invariance of the length of a maximal $E$-sequence. However, in view of 3.3 , it suffices to prove this for complete local rings. Since every complete local ring $R$ is the quotient of a regular local ring [4], we may assume that $R=T / \mathfrak{f}$ where $T$ is a regular local ring. Clearly $x_{1}, \cdots, x_{s}$ is a maximal $E$-sequence in $R$ if and only if one can find $y_{1}, \cdots, y_{s}$ in $T$ such that $\left(\mathfrak{l}, y_{i}\right) / \mathfrak{f}=x_{i}$, and such that $y_{1}, \cdots, y_{s}$ is a maximal $E$-sequence in $T$. Since $T$ is regular, we have by 2.3 that $s+\operatorname{hd}_{\mathrm{T}} E=\operatorname{dim} T$ if and only if $y_{1}, \cdots, y_{0}$ is a maximal $E$-sequence in $T$. Thererore, by our 
previous observation, $x_{1}, \cdots, x_{s}$ is a maximal $E$-sequence in $R$ if and only if $s+\operatorname{hd}_{\mathrm{T}} E=\operatorname{dim} T$. Thus any two maximal $E$-sequences in $R$ have length $s=\operatorname{dim} T-\mathrm{hd}_{\mathrm{T}} E$.

Proposition 3.5. If $R$ is a local ring, and $h d_{R} E<\infty$, then $h d_{R} E=\operatorname{codim} R$ if and only if $\mathfrak{m}$ belongs to (0) in $E$.

Proof. Suppose $\mathfrak{m}$ does not belong to $(0)$ in $E$. Then there is an $x \in \mathfrak{m}$ which is not a zero divisor of $E$ and thus $h_{\mathrm{R}} E / x E=1+\mathrm{hd}_{\mathrm{R}} E$. Thus by 1.6, $\operatorname{hd}_{\mathrm{R}} E \neq \operatorname{codim} R$.

Now suppose $\mathfrak{m}$ does belong to $(0)$ in $E$. Then, passing to completion, we have $\widehat{m}$ belongs to $(0)$ in $\hat{E}$. Let $\hat{R}=T / \mathfrak{l}$ where $T$ is a regular local ring. If we denote by $\overline{\mathfrak{m}}$ the maximal ideal of $T$, then $\overline{\mathfrak{m}}$ belongs to $(0)$ in $\hat{E}$, so that $\operatorname{hd}_{\mathbf{T}} \hat{E}=\operatorname{dim} T$. From the relationship

$$
\operatorname{hd}_{\mathrm{T}} E \leqq \operatorname{hd}_{\mathrm{T}} \hat{R}+\operatorname{hd}_{\mathrm{R}} \hat{E},
$$

we obtain

$$
\operatorname{dim} T-\operatorname{hd}_{\mathrm{T}} \hat{R}=\operatorname{codim} \hat{R} \leqq \operatorname{hd}_{\widehat{R}} \hat{E} .
$$

But, by $3.2, \operatorname{hd}_{\mathrm{R}} E=\mathrm{hd}_{\mathrm{R}} \hat{E}$, and $\operatorname{codim} R=\operatorname{codim} \hat{R}$, so that $\operatorname{codim} R \leqq \mathrm{hd}_{\mathrm{R}} E$. But the reverse inequality is given by 1.6 , so the proof is complete.

Proposition 3.6. Let $0 \rightarrow E^{\prime} \rightarrow E \rightarrow E^{\prime \prime} \rightarrow 0$ be an exact sequence of $R$-modules. If $\operatorname{codim}_{R} E^{\prime \prime}<\operatorname{codim}_{R} E$, then $\operatorname{codim}_{R} E^{\prime}=1+\operatorname{codim}_{R} E^{\prime \prime}$.

Proof. We may clearly assume that $R$ is a factor ring of a regular local ring $T$. In that case, we have $\operatorname{hd}_{\mathrm{T}} E<\mathrm{hd}_{\mathrm{T}} E^{\prime \prime}$, hence $\mathrm{hd}_{\mathrm{T}} E^{\prime \prime}=1+\mathrm{hd}_{\mathrm{T}} E^{\prime}$. But then $\operatorname{codim}_{T} E^{\prime}=1+\operatorname{codim}_{T} E^{\prime \prime}$ and since $\operatorname{codim}_{T} E=\operatorname{codim}_{R} E$ for all $R$-modules $E$, we obtain the desired result.

Using arguments similar to those used in establishing 2.3 , we obtain the following analogous result:

Theorem 3.7. Assume codim $R=n$ ( $R$ a local ring), $h d_{R} E<\infty$, and $x_{1}, \cdots, x_{8}$ is an E-sequence. Then we have

(a) $s+h d_{R} E \leqq n$,

(b) $s+h d_{R} E=n$ if and only if $x_{1}, \cdots, x_{s}$ is a maximal E-sequence.

COROLlary 3.8. Let $R^{\prime}$ be a factor ring of the local ring $R$, and $E$ an $R^{\prime}$ module. If $h d_{R} R^{\prime}$ and $h d_{R^{\prime}} E$ are finite, then

$$
h d_{R} E=h d_{R} R^{\prime}+h d_{R^{\prime}} E .
$$

By [3, XVI, Exercise 5] we have

$$
\mathrm{hd}_{\mathrm{R}} E \leqq \mathrm{hd}_{\mathrm{R}} R^{\prime}+\mathrm{hd}_{\mathrm{R}^{\prime}} E
$$

and so, in particular, hd $\mathrm{R}_{\mathrm{R}} E$ is finite. By 3.7, we have $\operatorname{codim}_{\mathrm{R}} E=\operatorname{codim} R-\mathrm{hd}_{\mathrm{R}} E$, 
$\operatorname{codim}_{R^{\prime}} E=\operatorname{codim} R^{\prime}-\mathrm{hd}_{\mathrm{R}^{\prime}} E, \operatorname{codim} R$-codim $R_{\mathrm{s}}=\mathrm{hd}_{\mathrm{R}} R^{\prime}$, which, combined with the fact that $\operatorname{codim}_{R} E=\operatorname{codim}_{R^{\prime}} E$, yields the desired result.

Proposition 3.9. Let $R$ be a (not necessarily local) ring, and let $\mathfrak{f}$ be an ideal of rank greater than zero. If $h d_{R} \mathfrak{f}<\infty$, then $\mathfrak{f}$ contains at least one nonzero divisor.

Proof. Suppose $\mathfrak{f}$ consists only of zero divisors. Then $\mathfrak{f}$ is contained in some prime $p$ belonging to (0), of rank greater than zero. Since rank $\mathfrak{f}>0$, and rank $R_{\mathfrak{p}} \mathfrak{f} \geqq$ rank $\mathfrak{f}$ in $R_{\mathfrak{p}}, R_{\mathfrak{p}} \mathfrak{f} \neq 0$. Now, $R_{\mathfrak{p}} \mathfrak{p}$ belongs to $(0)$ in $R_{\mathfrak{p}}$, so codim $R_{\mathfrak{p}}=0$. Therefore, by 1.6 , we have that hd $\mathrm{R}_{\mathrm{p}} R_{\mathfrak{p}} / R_{\mathfrak{p}} \mathfrak{f}=\infty$, since $R_{\mathfrak{p}} / R_{\mathfrak{p}} \mathfrak{f}$ is not projective. But then we have $\operatorname{hd}_{\mathrm{R}} R / \mathfrak{l}=\infty$, which is a contradiction.

4. Regular rings. In this section, we assume that $R$ is a commutative noetherian ring with identity element.

Definition. An ideal $\mathfrak{f}$ in $R$ is said to be regular if $R_{\mathfrak{p}}$ is a regular local ring for each prime $p$ belonging to $f$. $R$ is said to be regular if every ideal of $R$ is regular.

Proposition 4.1. $R$ is regular if and only if each maximal ideal in $R$ is regular.

Proof. The necessity follows from the definition. To prove sufficiency, we remark that it suffices to prove that $R_{\mathfrak{p}}$ is regular for every prime $\mathfrak{p}$. Therefore,

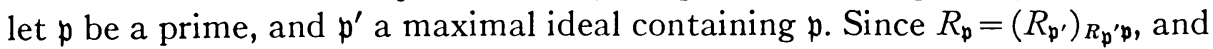
since $R_{\mathfrak{p}^{\prime}}$ is regular by hypothesis, the result follows from 2.14 .

Proposition 4.2. A regular integral domain $R$ is integrally closed.

Proof. Since $R=\bigcap_{\mathfrak{p}} R_{\mathfrak{p}}$ where $\mathfrak{p}$ runs through all maximal ideals, and since each $R_{\mathfrak{p}}$, being regular, is integrally closed, $R$ is the intersection of integrally closed rings. But then $R$ is integrally closed.

Proposition 4.3. $R$ is the direct sum of a finite number of integral domains if and only if $R_{\mathfrak{p}}$ is an integral domain for every prime $\mathfrak{p}$.

Proof. Suppose $R_{\mathfrak{p}}$ is an integral domain for every prime $\mathfrak{p}$. Let (0) $=\mathfrak{q}_{1} \cap \cdots \cap \mathfrak{q}_{n}$ be a normal decomposition of $(0)$, and let $\mathfrak{p}_{1}, \cdots, \mathfrak{p}_{n}$ be the primes belonging to $\mathfrak{q}_{1}, \cdots, \mathfrak{q}_{n}$ respectively. We will show that $\mathfrak{q}_{i}=\mathfrak{p}_{i}$ for all $i$, and hence each $p_{i}$ is minimal, due to the irredundancy of the decomposition. Suppose $\mathfrak{q}_{i} \neq \mathfrak{p}_{i}$. Then $R_{\mathfrak{p}_{\mathfrak{i}}}$ contains zero divisors, contrary to assumption. Thus $q_{i}=p_{i}$ for all $i$.

If $n=1$, we are finished. Suppose, then, that $n>1$. If $\left(\mathfrak{p}_{i}, \mathfrak{p}_{j}\right) \neq R(i \neq j)$, let $\mathfrak{p}$ be a proper prime ideal containing $\left(\mathfrak{p}_{i}, \mathfrak{p}_{j}\right)$. Then in $R_{\mathfrak{p}}$ we have zero divisors, again contrary to assumption. Thus, for all pairs $(i, j), i \neq j$, we have $\left(\mathfrak{p}_{i}, \mathfrak{p}_{j}\right)$ $=R$. Applying the Chinese Remainder Theorem, we have $R \approx R / p_{1}+\cdots$ $+R / \mathfrak{p}_{n}$ (direct sum).

Now let $R=R_{1}+\cdots+R_{n}$ (direct sum) with each $R_{i}$ an integral domain. Let $e_{i}$ denote the $i$ th natural base elements and let $\mathfrak{p}_{i}$ be the ideal generated 
by all $e_{j}, j \neq i$. It is easy to see that each $\mathfrak{p}_{i}$ is a prime. Let $\mathfrak{p}$ be a proper prime in $R$. Then since $\sum e_{i}=1, p$ does not contain $e_{i}$ for some $i$. If $\mathfrak{p}$ does not contain $\mathfrak{p}_{i}$, then there is an $e_{j}, j \neq i$, such that $e_{j} \notin p$. But this is impossible since $e_{i} e_{j}=0$ for $j \neq i$. Thus every prime contains some $\mathfrak{p}_{i}$. Furthermore, if $\mathfrak{p} \supset \mathfrak{p}_{i}$, then $\mathfrak{p}=\mathfrak{p}_{i}+\mathfrak{p} \cap R_{i}$ (direct sum). Therefore $R_{\mathfrak{p}} \approx\left(R_{i}\right)_{\mathfrak{p} \cap R_{\mathfrak{i}}}$ and hence $R_{\mathfrak{p}}$ is an integral domain for each $\mathfrak{p}$.

CoRollary 4.4. A regular ring is the direct sum of regular integral domains.

Proposition 4.5. A ring $R$ of finite global dimension is regular.

Proof. For each prime $\mathfrak{p}, R_{\mathfrak{p}}$ has finite global dimension, hence is regular.

Lemma 4.6. If $E$ is an R-module, then $h d_{R} E=\sup \left(h d_{R_{\mathfrak{p}}}\left(R_{\mathfrak{p}} \otimes_{R} E\right)\right)$ where $\mathfrak{p}$ runs through all maximal ideals of $R$. Hence gl. $\operatorname{dim} R=\sup .\left(\mathrm{gl} . \operatorname{dim} R_{\mathfrak{p}}\right)$, where $\mathfrak{p}$ runs through all maximal ideals of $R$.

Proof. The first statement is found in [3, VII, Exercise 11]. To prove the second, we again make use of [1, Theorem 1$]$, and restrict our attention to the modules $R / \mathfrak{l}$, where $\mathfrak{l}$ is any ideal of $R$. Thus we have gl. $\operatorname{dim} R$ $=\sup \left(\operatorname{hd}_{\mathrm{R}} R / \mathfrak{f}\right)=\sup \left(\sup \left(\operatorname{hd}_{\mathrm{Rp}}\left(R_{\mathfrak{p}} \otimes_{R} R / \mathfrak{f}\right)\right)\right) \leqq \sup \left(\mathrm{gl}\right.$. $\left.\operatorname{dim} R_{\mathfrak{p}}\right)$. On the other hand gl. $\operatorname{dim} R \geqq \sup \left(h_{\mathrm{R}} R / \mathfrak{p}\right)=\sup \left(\mathrm{gl} . \operatorname{dim} R_{\mathfrak{p}}\right)$, and so we are done.

TheOREM 4.7. In a regular ring $R, g l . \operatorname{dim} R=\operatorname{dim} R$.

Proof. In a regular ring, gl. $\operatorname{dim} R_{\mathfrak{p}}=\operatorname{dim} R_{\mathfrak{p}}$. Since $\operatorname{dim} R=\sup \left(\operatorname{dim} R_{\mathfrak{p}}\right)$, 4.6 gives us the result.

COROLlary 4.8. A ring of finite dimension is regular if and only if it has finite global dimension.

Proposition 4.9. Let $R$ be the quotient of a regular ring. Then $R$ satisfies the saturated chain condition (see 2.11).

Proof. Let $R=T / \mathfrak{f}$, where $T$ is regular ring. Let $p_{1} \subset p_{2}$ be two primes in $R$, and suppose that $\mathfrak{p}_{2}$ is the image of $\mathfrak{p}^{\prime}$ in $T$. Then $R_{\mathfrak{p}_{2}} \approx T_{\mathfrak{p}^{\prime}} / T_{\mathfrak{p}^{\prime}} \mathfrak{p}$. Hence $R_{\mathfrak{p}_{2}}$ is the quotient of a regular local ring. Since there is a 1-1 correspondence between primes of $R$ contained in $\mathfrak{p}_{2}$ and primes in $R_{\mathfrak{p}_{2}}$, the result follows from 2.10 .

Proposition 4.10. If $\mathfrak{t}$ is a regular ideal in $R$, then $h d_{R} R / \mathfrak{l} \geqq$ rank $\mathfrak{p}$, where $\mathfrak{p}$ is any prime belonging to $\mathfrak{l}$. Thus, if $h d_{R} R / \mathfrak{l}=$ rank $\mathfrak{l}$, then $\mathfrak{l}$ is unmixed.

Proof. We know that if $\mathfrak{p}$ is a prime belonging to $\mathfrak{l}$, then $h_{\mathrm{R}} R / \mathfrak{f}$ $\geqq \mathrm{hd}_{\mathrm{Rp}}\left(R_{\mathfrak{p}} \otimes_{R} R / \mathfrak{f}\right)=\mathrm{hd}_{\mathrm{Rp}} R_{\mathfrak{p}} / R_{\mathfrak{p}} \mathfrak{f}$. Since $R_{\mathfrak{p}} \mathfrak{p}$ belongs to $R_{\mathfrak{p}} \mathfrak{l}$ in $R_{\mathfrak{p}}$, we have $\operatorname{hd}_{\mathrm{R}} R / \mathfrak{f} \geqq$ gl. $\operatorname{dim} R_{\mathfrak{p}}=\operatorname{dim} R_{\mathfrak{p}}=$ rank $\mathfrak{p}$.

PROPOSITION 4.11. Let $R$ be a regular ring, and $\mathfrak{t}=\left(x_{1}, \cdots, x,\right)$ an ideal of rankr. Then $x_{1}, \cdots, x_{r}$ is an $R$-sequence. Hence $\operatorname{hd}_{\mathrm{R}} R /\left(x_{1}, \cdots, x_{r}\right)=r$, and $\mathfrak{l}$ is unmixed. 
Proof. Since $R$ is a regular ring, it satisfies the saturated chain condition by 4.9 . Hence, applying 2.11 , we see that $\left(x_{1}, \cdots, x_{r-1}\right)$ has rank $r-1$. Suppose the proposition true for $r-1$. Then, as in 2.12 , we have that $x_{1}, \cdots, x_{r}$ is an $R$-sequence. Since rank $\left(x_{1}, \cdots, x_{r}\right)=r$, we know that hd $\mathrm{R}_{\mathrm{R}} R /\left(x_{1}, \cdots, x_{r}\right)$ $\geqq r$. Hence we need only show that $\operatorname{hd}_{\mathrm{R}} R /\left(x_{1}, \cdots, x_{r}\right) \leqq r$. However, this follows from the general fact that if $E$ is an $R$-module, and $x$ is an $E$-sequence, then $\operatorname{hd}_{\mathrm{R}} E / x E \leqq 1+\mathrm{hd}_{\mathrm{R}} E$. For from the exact sequence

$$
0 \rightarrow E \stackrel{f}{\rightarrow} E \rightarrow E / x E \rightarrow 0
$$

where $f$ is multiplication by $x$, we obtain the exact sequence

$$
\cdots \rightarrow \operatorname{Ext}_{R}^{n}(E, C) \rightarrow \operatorname{Ext}_{R}^{n+1}(E / x E, C) \rightarrow \operatorname{Ext}_{R}^{n+1}(E, C) \rightarrow \cdots
$$

for every $R$-module $C$. Hence if $\operatorname{Ext}_{R}^{p}(E, C)=0$ for all $p>s$, then $\operatorname{Ext}_{R}^{p+1}(E / x E$, $C)=0$ for all $p>s$.

Now we must prove the proposition for $r=1$.

Let $k=(x)$ be of rank 1. If $x$ were a zero divisor, it would be contained in some prime belonging to $(0)$. But $0=h_{\mathrm{R}} R /(0)=\sup$ rank $\mathfrak{p}$ where $\mathfrak{p}$ runs through all primes belonging to (0). Hence all the primes belonging to (0) are of rank zero, and therefore cannot contain an ideal of rank one.

Since $x$ is not a zero divisor, $\operatorname{hd}_{\mathrm{R}} R /(x) \leqq 1$. But $\mathrm{hd}_{\mathrm{R}} R /(x) \geqq$ sup rank $p$ where $p$ belongs to $x$. Hence $\operatorname{hd}_{\mathrm{R}} R /(x) \geqq 1$, thus $\operatorname{hd}_{\mathrm{R}} R /(x)=1$, and rank $p=1$ for all $\mathfrak{p}$ belonging to $(x)$. Thus our proposition is established for $r=1$, hence for all $r$.

Proposition 4.12. In a regular integral domain $R$ of dimension less than or equal to two, all minimal primes are invertible.

Proof. Let $\mathfrak{p}^{\prime}$ be a minimal prime, i.e., a prime of rank 1 . Then $h_{\mathrm{R}} R / \mathfrak{p}^{\prime}$ $=\sup \left(\mathrm{hd}_{\mathrm{R}_{\mathfrak{p}}} R_{\mathfrak{p}} / R_{\mathfrak{p}} \mathfrak{p}^{\prime}\right)$ where $\mathfrak{p}$ runs through all maximal ideals containing $\mathfrak{p}^{\prime}$. Since all maximal primes have rank $\leqq 2, R_{\mathfrak{p}} / R_{\mathfrak{p}} \mathfrak{p}^{\prime}$ has codimension 1 as an $\mathrm{R}_{\mathfrak{p}}$-module, hence $\mathrm{hd}_{\mathrm{Rp}} R_{\mathfrak{p}} / R_{\mathfrak{p}} \mathfrak{p}^{\prime}=1$. Thus $\mathrm{hd}_{\mathrm{R}} R / \mathfrak{p}^{\prime}=1, \mathrm{hd}_{\mathrm{R}} \mathfrak{p}^{\prime}=0, \mathfrak{p}^{\prime}$ is projective and hence invertible [3, VII, 3.2].

Proposition 4.13. A ring is hereditary if and only if it is the direct sum of a finite number of Dedekind rings.

Proof. A ring $R$ is hereditary if and only if $\operatorname{gl} . \operatorname{dim} R \leqq 1$. Thus the result follows from 4.4, and the fact that if $R=R_{1}+\cdots+R_{n}$ (direct sum), then gl. $\operatorname{dim} R=\sup$ gl. $\operatorname{dim} R_{i}$.

Appendix (added in proof). Some recent work of the authors (to appear) has shown that 3.4 can be proved without using the structure theorems of Cohen. These techniques also yield the result of 2.13 for an arbitrary local ring. 
It can also be shown that 3.9 holds without the hypothesis that rank $\mathfrak{l}>0$.

\section{BIBLIOGRAPHY}

1. M. Auslander, On the dimension of modules and algebras, III. Global dimension, Nagoya Math. J. vol. 9 (1956).

2. M. Auslander and D. A. Buchsbaum, Homological dimension in noetherian rings. Proc. Nat. Acad. Sci. U.S.A. vol. 42 (1956).

3. H. Cartan and S. Eilenberg, Homological algebra, Princeton University Press, 1956.

4. I. S. Cohen, On the structure and ideal theory of complete local rings, Trans. Amer. Math. Soc. vol. 59 (1946) pp. 54-106.

5. W. Grobner, Moderne algebraische Geometrie, Berlin, Springer, 1949.

6. W. Krull, Dimensiontheorie in Stellenringen, J. Crelle vol. 179 (1938).

7. D. G. Northcott, Ideal theory, Cambridge University Press, 1953.

8. P. Samuel, Commutative algebra (Notes by D. Hertzig), Cornell University, 1953.

9. J.-P. Serre, Faisceaux algébriques cohérents, Ann. of Math. vol. 61 (1955).

10. - Sur la dimension des anneaux et des modules noetherriens, Proceedings of the International Symposium on Algebraic Number Theory, Tokyo and Nikko, 1955, Science Council of Japan, Tokyo, 1956.

UNIVERSITY OF Michigan, ANN ARbor, Mich.

University of Chicago, Chicago, Ill. 